Páez V, J.E. (2019). Prevenir el consumo de tabaco en el ciberespacio escolar de la ciudad de Pereira-Colombia. Plumilla Educativa, 24(2), 85-107. DOI 10.30554/p.e.2.3576.2019

\title{
Prevenir el consumo de tabaco en el ciberespacio escolar de la ciudad de Pereira-Colombia
}

Julián Enrique Páez Valdez ${ }^{1}$

\section{Resumen}

El presente artículo estudia la relación entre herramientas tecnológicas y comunicación en salud, con el fin de comprender cómo los jóvenes utilizan los dispositivos digitales y revisar la efectividad que tendrían estos espacios para disminuir el consumo de tabaco. El trabajo utilizó un método mixto de encuestas, grupos focales y fotografía participativa, que permitió comprender cómo las redes sociales se han convertido en un espacio público digital juvenil y por consiguiente, una herramienta útil para transmitir campañas de promoción y prevención en temas de salud y políticas públicas. Los jóvenes se ven enfrentados a múltiples espacios de representación, visibilización e intercambio en línea, llevando a los jóvenes a evaluar su rol como ciudadanos digitales en múltiples ciberespacios, es por esta razón que las campañas de comunicación colaborativa y transmedia pueden ser la solución para disminuir la percepción positiva hacia el tabaco y la repetición de pares.

Palabras clave: Herramientas tecnológicas; Redes sociales digitales; Campañas de comunicación en salud; Consumo de tabaco; Ciberespacio escolar

1 Julián Enrique Páez Valdez. Magister en Ciencias Humanas, mención información y comunicación de la Université Sorbonne Nouvelle; Doctorando en Ciencias de la Información y de la Comunicación, Université Sorbonne Paris Cité; Becario Cofund Inspire - Unión Europea y doctorando contractual Université Sorbonne Nouvelle Paris 3; Docente investigador del programa de Comunicación Social Periodismo de la Universidad Católica de Pereira; Líder del grupo de investigación: Comunicación, Educación y Cultura - COL0031566; Orcid: https://orcid.org/0000-0001-5874-5767. Correo electrónico: julian.paez@ucp.edu.co 


\section{Prevent the use of cigarette in school cyberspace of the city of Pereira Colombia}

Abstract

This article studies the relation between technological tools and communication processes in health, with an aim to understand how young people use digital communication tools and thus review the effectiveness which those spaces will have as comparative tools in order to diminish the consumption of cigarette. The following work used a mixed method of surveys, focus groups and participatory photography which enabled us to understand how digital social networks have turned into a constant digital public space and consequently become a useful tool in order to provide promotion and prevention campaigns on topics of health. Young people are faced with numerous spaces of representation and exchange which currently gives young people the opportunity to assess their role as digital citizens in multiple cyberspaces. It is for this reason that collaborative and transmedia communication campaigns can be the solution to reduce the positive perception towards cigarette and the repetition of peers.

Key words: Technological tools; Digital social networks; Health communication campaigns; Cigarette consumption; school cyberspace.

\section{Prevenir o consumo de tabaco na} ciberespaço escolar da cidade de Pereira Colombia

\section{Resumo}

Este artigo estuda a relação entre ferramentas tecnológicas e comunicação em saúde, a fim de entender como os jovens usam dispositivos digitais e revisar a eficácia que esses espaços teriam para reduzir o consumo de tabaco em jovens. $O$ trabalho utilizou um método misto de pesquisas, grupos focais e fotografias participativas que nos permitiram entender como as redes sociais se tornaram um espaço público digital juvenil e, portanto, uma ferramenta útil para transmitir campanhas de promoção e prevenção sobre questões políticas e de saúde público. Os jovens enfrentam múltiplos espaços de represen- 
tação, visibilidade e intercâmbio on-line, levando os jovens a avaliar seu papel como cidadãos digitais em vários ciberespaços. É por esse motivo que as campanhas de comunicação colaborativa e transmídia podem ser a solução para reduzir a percepção positiva em relação ao tabaco e a repetição de colegas.

Palavras-chave: Ferramentas tecnológicas; Redes sociais digitais; Campanhas de comunicação em saúde; Consumo de tabaco; ciberespaço escolar.

\section{Introducción ${ }^{2}$}

El presente artículo pretende detectar cómo otros espacios públicos de intercambio informativo, argumentativo y narrativo, pueden ser útiles para disminuir el consumo de tabaco y los diferentes tipos de cáncer derivados de esta práctica, en especial, estudiando los espacios públicos digitales, como los que se derivan del uso de las redes sociales digitales y las aplicaciones web, ampliamente conocidas en la comunidad juvenil.

El consumo de tabaco en jóvenes es largamente estudiado, se ha logrado determinar por ejemplo que la edad promedio de inicio del consumo de tabaco en colombianos es cercano a los 12 años, así como se ha logrado detectar que los jóvenes de Colombia están expuestos entre un $40 \%$ y un $60 \%$ a espacios con humo de tabaco, aumentando el riesgo de tomar el hábito de fumar en un 30\% (León y Bonilla, 2011).

Por otra parte, (Pérez y Pinzón-Pérez, 2005) (Pardo y Piñeros, 2010) han logrado evidenciar que cerca del $50 \%$ de los jóvenes estudiantes del país han recibido información sobre los riesgos de consumir tabaco, sin embargo, como lo afirman los mismos autores, es posible que muchos de esos consumos se den gracias a la poca efectividad de dichas campañas en espacios tradicionales, así como la transmisión a partir de modelos de aprendizaje social entre familiares y amigos, lo que permite reafirmar la importancia de estudiar espacios alternativos para emitir estas campañas.

2 El presente artículo es un resultado de investigación derivado del proyecto titulado: Photoboy, una herramienta tecnológica para la promoción y prevención de diferentes tipos de cáncer causados por el consumo de tabaco, en jóvenes de la ciudad de Pereira. Financiado por la Universidad Católica de Pereira / DII-015-004 
Es por esta razón que el presente trabajo de investigación se basó en estudiar las actitudes previas y estados de contemplación de los jóvenes hacia el tabaco, para finalmente contrastar cómo dichas actitudes y estados se podían modificar con la implementación de estrategias de comunicación digital basadas en la fotografía participativa, el uso pedagógico de medios y la creación colaborativa transmedia, mediante el proyecto de intervención digital denominado Photoboy, el cual buscó enviar mensajes de prevención a los jóvenes a través del ciberespacio escolar que posibilita el uso de las redes sociales digitales como Facebook.

\section{Referente teórico}

En la actualidad los medios de comunicación son utilizados como canales comunicativos por donde se introducen mensajes de tipo informativo o comunicativo a los miembros de la sociedad, es el caso del sector salud, que no se ha visto alejado de esta realidad y ha intentado por los canales tradicionales como la televisión, la radio y la prensa, promocionar hábitos de vida saludable, así como prevenir muchas de las enfermedades que tenemos en la actualidad y que sin duda alguna, se vuelven de discusión pública y por consiguiente se manejan no solo en la agenda informativa, sino también en la agenda política de la mayoría de países o regiones (Mosquera 2013), (Gerhard Maletzke, 1976)

En este orden de ideas, estudiar la relación entre medios masivos de comunicación y salud es importante, pues siempre estamos expuestos a este tipo de mensajes, sin embargo, pocas veces estudiamos su efectividad.

\section{El rol de los medios, un acercamiento a las campañas tradicionales de prevención}

Los usos mediáticos (Lull, 1978), (Mcquail et al., 1972) nos permiten identificar las funciones que deben tener los medios, y por consiguiente, los mensajes que allí circulan. Es así como podemos identificar tres funciones básicas de cualquier medio de comunicación, como espacio de circulación de la opinión pública: informar, entretener y educar. Los cuales se calculan en los tres procedimientos básicos de la construcción comunicativa: la producción, la difusión y la recepción, es allí donde se ve la importancia de estudiar los usos sociales que generan los medios de comunicación, ya que, en el caso de esta investigación, las campañas mediáticas se crean para públicos objetivos, pero pocas veces se evalúan los resultados de estas, desde los mismos usos que le dan los usuarios a los soportes desde donde reciben los mensajes. 
Páez V, J.E. (2019). Prevenir el consumo de tabaco en el ciberespacio escolar de la ciudad de Pereira-Colombia. Plumilla Educativa, 24(2), 85-107. DOI

Es importante comprender cómo el uso de herramientas tecnológicas en los espacios públicos digitales escolares se puede comprender estudiando los acercamientos teóricos de autores como (Lull, 1978) quien estudió la manera en la que las personas usan los contenidos mediáticos que circulan en cualquier soporte comunicativo. Sin embargo, el desarrollo mayor se dio con (Mcquail et al., 1972), quienes aumentaron a este acercamiento inicial una perspectiva mayor sobre las gratificaciones y sobre todo las motivaciones que llevan a las personas a usar los medios.

En el caso de (Lull, 1978) las personas usan los medios y reciben dichos mensajes para dos cosas, en un primer momento, un uso estructural, que equivale a la presencia del medio y del mensaje en espacios públicos o privados, pero que no garantizan un buen proceso de recepción informativa o comunicativa. Para el caso de este proyecto de investigación, es relevante decir que los estudiantes dentro de sus espacios públicos tradicionales y digitales, reciben y están usando contenidos de manera estructural, ya que muchos de los mensajes no son lo suficientemente interesantes para llamar la atención o simplemente los reciben cuando usan sus dispositivos en etapas inactivas, por ejemplo: muchos de los contenidos gráficos de Instagram, red social que hace parte de ese ciberespacio escolar en la actualidad, se reciben cuando el estudiante está en clases que no le generan interés y el objetivo de su uso es simplemente ser un acompañamiento a ese momento de aburrimiento.

Sin embargo, (Lull,1978) se acercó a los usos relacionales, es decir, a esos que generan un proceso de significación en los usuarios, aquellos que después de la etnografía y observación directa ejecutada por el investigador, lograron evidenciar que facilitan la comunicación, logran identificar la pertenencia o exclusión a los grupos sociales (Anzieu y Zimmermann, 1997), posibilitan el aprendizaje social y demuestran las competencias o dominios. Esta serie de usos mediáticos pueden demostrar también la efectividad de los mensajes comunicativos, ya que el soporte en sí tiene una carga de atracción inicial con el usuario, sin embargo, es el mensaje o contenido comunicativo que allí se vehicula, el que tiene la mayor carga motivacional dentro de la relación existente entre las personas y los contenidos mediáticos en diversos soportes.

Acercando la teoría anterior al objeto de estudio evidente en este artículo, es importante saber que muchos de los contenidos comunicativos en salud se acercan a soportes bastante motivadores como lo son la televisión y la internet, sin embargo, los mensajes presentes en los mismos no son lo bastantes llamativos en los jóvenes escolares, ya que no se convierten en temas de discusión en esos espacios de intercambio tradicionales y virtuali- 
zados. Tal es el caso de las campañas que se evaluaron con este proyecto y que podríamos listar de la siguiente manera

- La decisión está en tus manos: campaña de comunicación de Oncólogos de Occidente

- Conversatorios de cáncer infantil: campaña de prevención de Oncólogos de Occidente

- Generación somos +: del Gobierno colombiano en cabeza del Ministerio de la Protección Social

- Que no te metan los dedos a la boca: de la Liga colombiana contra el cáncer

- QuiteNow: app para dejar de fumar

- Cessation Nation: app para la prevención del consumo del tabaco.

Como se evidencia en la presente selección se utilizaron campañas que circulan por espacios mediáticos tradicionales como la televisión, la radio y el papel, pero también otras que usan los medios digitales para su distribución, sin embargo se evaluó sobre todo la recepción de dichas campañas, ya que la función fundamental de un medio es lograr una etapa de receptividad basada en un uso estructural (Lull, 1978) que ilustren las experiencias, generen agendas de conversación, mantengan las relaciones o creen conflictos, permitan la toma de decisiones, posibiliten en diferentes grados la modelización del comportamiento, realicen procesos de legitimación, refuercen los roles, faciliten la discusión, entre otros.

Sin embargo, hablar de los usos sociales de los medios para comprender la efectividad de las campañas de promoción y prevención en salud, es importante reconocer 4 niveles de usabilidad según (Mcquail et al., 1972) y que se basan en el entretenimiento, las relaciones personales, la identidad personal y la vigilancia, los cuales responden a esa identificación de factores sociales generalizados y que finalmente se convierten en los aspectos de obligatoriedad que deberían incluir todos los mensajes comunicativos, con el fin de llegar a un nivel de recepción claro y apropiación evidenciada por parte de los usuarios.

Desde el año 1986, la Organización Mundial de la Salud (WHO, 1986) determinó que los medios de comunicación masiva e información son espacios vitales para transmitir campañas para la promoción de la salud, enfocándose sobre todo en cómo dichos espacios pueden prevenir conductas de riesgo e influenciar en las decisiones personales o grupales que disminuyan las enfermedades en el mundo. "La comunicación en salud abarca el estudio y el uso de estrategias de comunicación para informar e influenciar decisiones individuales y comunitarias que mejoren la salud". (Mosquera y Mateus 2003, p.1) 
Páez V, J.E. (2019). Prevenir el consumo de tabaco en el ciberespacio escolar de la ciudad de Pereira-Colombia. Plumilla Educativa, 24(2), 85-107. DOI

La encuesta mundial de tabaquismo en jóvenes (WHO, 2007) afirma que cerca de 17000 muertes anuales en Colombia se deben al consumo de tabaco, lo que lo convierte en uno de los problemas más graves en salud pública, junto con otras enfermedades no transmisibles como lo son la obesidad ligada a la falta del ejercicio y la mala nutrición.

La carta de Ottawa responde además a la necesidad de frenar la comercialización masiva y libre de un producto tan nocivo como el tabaco, ya que la industria tabacalera gasta anualmente millones de dólares comercializando sus productos en diversos países a través de 3 estrategias fundamentales: la publicidad, la promoción y los patrocinios, los cuales se presentan como herramientas que tienden a mostrar los productos que contienen tabaco como poco invasivos y peligrosos para la salud. Es importante reconocer que cada país debe aplicar sus propias políticas para la promoción y prevención, es por esta razón que en el caso de Colombia se prohíben los patrocinios tabacaleros a cualquier evento deportivo o cultural, así como las publicidades en cualquier medio de comunicación, la venta al menudeo, las campañas multimedia, así como la promoción mediante la distribución visible en tiendas y grandes superficies. Ley 1335 de 2009 (Congreso de la República de Colombia, 2009) y la Resolución 2010-380-001136-4 de la (Comisión Nacional de Televisión, 2010)

Es importante reconocer además que el consumo de tabaco se relaciona directamente con el cáncer, por consiguiente, muchos de los esfuerzos que se ejecutan para evitar su promoción abierta están dados en disminuir las muertes causadas por esta enfermedad, es por esto que el número 19 de la Colección monográfica para la lucha antitabaco, publicado por el National Cáncer Institute (NCl, National Cáncer Institute 1991), se hace énfasis en tres eventos importantes, primero: el desarrollo acelerado de los medios y sus tecnologías, segundo: el aumento del tabaquismo como fenómeno social y más recientemente la comprensión de la relación existente entre tabaco y cáncer.

Según el Comité Nacional Contra el Tabaquismo de Francia (Comité National Contre le Tabagisme, 2016), se necesita una difusión regular de campañas de prevención en los medios con el fin de informar al público en general sobre los efectos del consumo de tabaco, siendo entonces el artículo 12 de la convención general Antitabaco en Colombia (CCLAT, 2009), en donde se afirma que se debe luchar contra la publicidad propia de las empresas tabacaleras, hacer evolucionar y mejorar las percepciones de la población con respecto a la información del tabaco, así como "cambiar la imagen del tabaco, motivar a los fumadores a disminuir o parar el consumo, incitar a los no fumadores a seguirlo siendo, reducir el consumo en la población juvenil, 
así como hacer que la sociedad hable del tema" (CCLAT, 2009), sin embargo, todas estas estrategias de prevención se desarrollan en espacios mayores que podemos llamar esferas públicas y donde se garantiza la pluralidad informativa de la opinión pública. En el caso de los jóvenes, estos espacios de intercambio se han virtualizado y la mayor parte de conversaciones juveniles se dan en línea, a través de plataformas como Facebook, Instagram, YouTube o WhatsApp.

\section{Opinión pública y espacio público digital escolar}

Históricamente, los espacios públicos han sido estudiados desde la arquitectura y las ciencias urbanísticas, cómo esos lugares físicos compartidos por los pobladores de asentamientos urbanos, sin embargo, es de vital importancia empezar por comprender cómo los espacios públicos pueden ser abstractos y estudiados desde las ciencias sociales.

Para comprender el fenómeno de los espacios públicos digitales, o esferas públicas digitales, como se le conoce en Norte América, es importante mencionar una serie de autores clave como lo son (Fraser, 1990) (Habermas et al., 1981), (Champagne, 1995), (Benkler, 2006), entre otros estudiosos de la sociología de la comunicación, quienes a partir de sus desarrollos teóricos nos han llevado a concluir que "los espacios públicos normativos, que son diferentes a los espacios públicos geográficos y espaciales, se caracterizan por convertirse en lugares abstractos en los cuales se configuran procesos de discusión, intercambio y deliberación de los asuntos de interés para la sociedad" (Valdez J-P 2016, P. 31)

Un espacio público está compuesto por individuos, instituciones y sobre todo, canales de comunicación, por donde se transmiten los mensajes propios que se intercambian entre los medios, social y culturalmente similares, creando así un territorio de discusión, mayoritariamente político, sobre los asuntos de su ciudad, región o comunidad. Teniendo en cuenta esta premisa es relevante decir que son los espacios públicos los encargados de construir la opinión pública a través de las estrategias informativas o comunicativas existentes y puestas en marcha por los públicos, generando así temas de discusión que trascienden la esfera personal y se conviertan de interés público.

Podríamos decir entonces que el concepto de espacio público como lugar de circulación de las ideas nace con las ágoras griegas, ya que era allí donde se debatían los temas de las polis antiguas y que con el transcurso de la historia se han transformado, pasando de espacio cerrados y limitados, a escenarios abiertos gracias al uso de los medios masivos de comunicación y de internet. 
Páez V, J.E. (2019). Prevenir el consumo de tabaco en el ciberespacio escolar de la ciudad de Pereira-Colombia. Plumilla Educativa, 24(2), 85-107. DOI

Un ejemplo de mediatización en salud es la que se produce cuando grandes epidemias afectan a diversos países, más recientemente se vio como la crisis del ébola, la cual afectó a diversos países africanos, e incluso de otros continentes, estuvo como tema de discusión en los medios y por consiguiente como evento relevante en el espacio público, en este caso, global. Del mismo modo hemos visto esos procesos de mediatización en otras epidemias como el Zika, AH1N1, entre otras, las cuáles llegan al espacio público general por su condición de global, y más hoy en día, que la sociedad está interconectada y que los medios dejaron de ser locales para convertirse en globales. Según Suau-Gomila et al. (2017) en el caso de España "la conversación en Twitter sobre el Ébola no tuvo un carácter informativo, fue eminentemente emocional" $(p, 130)$, en un estudio de más de 1 millón de mensajes en la plataforma, así como 39 medios de comunicación durante la crisis del ébola en España durante 2014, se dieron cuenta que la muerte de más de 11 mil personas en el mundo entre 2014 y 2016 se convirtió en un tema de discusión ciudadana global y ampliado por el efecto refractor de las redes sociales digitales (Rieder, 2012).

Según Salván e Izquierdo (2014), la comunicación social que se hizo de la crisis del ébola pasó por una primera etapa de crisis, una segunda de alarma sanitaria y una final de desinformación, la cual llevó a que múltiples ciudadanos y autoridades nacionales, sobredimensionaran la crisis en territorios apartados de la problemática como España. No obstante, América Latina no ha estado alejada de los problemas de mediatización en temas de salud, ya que el virus del Zika se convirtió en un desafío para la salud pública de la región, pero también para los medios de comunicación, quienes pocas veces reconocen el impacto de la cobertura mediática y las políticas de framing y priming que realizan de estos hechos (Entman, 1993). (Scheufele y Tewksbury, 2006). Según Caylà et al. (2016), para el caso del Zika, la cobertura mediática se concentró en hablar de los casos de microcefalia en mujeres embarazadas, dándole un impacto mediático, que en ocasiones fue alarmista y buscaba ganar audiencias.

Los problemas de salud ascienden fácilmente a los espacios públicos, no obstante, no pasa lo mismo con las campañas de comunicación que buscan prevenir enfermedades o promocionar hábitos de vida saludable. Para el caso del tabaco, las campañas de comunicación deben lograr que no solo se hable de ellas, sino que permitan ascender a la esfera pública, debido a su condición de problemática. El mayor objetivo de este tipo de campañas es que los receptores de dichos mensajes repliquen el contenido y generen espacios de discusión mayor. En conclusión, estaríamos diciendo que las cam- 
pañas actualmente provocan reacciones particulares y no públicas, lo que lleva a que las mismas nunca se posicionan en grupos poblacionales como temas de discusión.

Es respondiendo a este tema que se propone que las campañas comunicativas para promocionar aspectos ligados a la salud deben contener las siguientes características:

- Deben ser reticulares y no olvidar la idea de glocalización de las personas de hoy en día, tal cual lo expresa (Beck, 1998).

- A pesar de que la salud tiende a ser un asunto particular, los mensajes no deberían ser construidos exclusivamente para llegar de manera individual, si tomamos el ejemplo del tabaco, es una enfermedad que mayoritariamente afecta a cada individuo (problemas de cáncer, entre otros), pero esto no la exime las consecuencias públicas que deriva su consumo.

- Los mensajes deben tocar las fibras individuales, pero deben ser capaces de afectar a grupos poblacionales y, por consiguiente, generar discusiones capaces de llegar a los espacios públicos a los que pertenecen los receptores de los contenidos.

- Para que un tema sea relevante para la sociedad se debe evidenciar cómo afecta lo público y no solo lo particular, es por esta razón que se deberían ensayar campañas que involucren a terceros, ejemplo: en las cajetillas dejar de mostrar consecuencias individuales, para empezar a mostrar problemas sociales, que logren a los que no están cercanos al problema evidenciar que en algún momento podrían ser afectados.

- Narrativamente la sociedad de hoy en día es transmedia, es decir, navega por múltiples soportes y plataformas, por consiguiente, la narrativa de los mensajes debería pensarse para que el usuario esté en constante cercanía con la idea informativa que se le transmite.

Herramientas tecnológicas en ciberespacios escolares como estrategia de prevención

Para el caso de los ciberespacios escolares, es fundamental comprender que muchas de las deficiencias de las herramientas tecnológicas propuestas es que no responden a estas cuatro características. Mediante un juego de realidad alternativa ( $A R G$ ), según la teoría transmedia de (Scolari, 2013), se puede lograr entretener a un estudiante e informarle los riesgos de enfermedades y así prevenir muchas actitudes que los llevan a desarrollar dichos problemas de salud. La mayoría de esos juegos no han pensado en cómo llevar a dicho usuario, no solo a reconocer la estrategia como un elemento de entretenimiento, sino también como uno que apoye sus 
Páez V, J.E. (2019). Prevenir el consumo de tabaco en el ciberespacio escolar de la ciudad de Pereira-Colombia. Plumilla Educativa, 24(2), 85-107. DOI

relaciones personales, lo lleven a esculpir su identidad personal y le promuevan una serie de vigilancias sociales sobre lo que se debe o no hacer en dichos casos.

Un estudio realizado sobre el nivel de digitalización de los colombianos (DANE, 2013), demuestra que 8 de cada 10 colombianos acceden a internet, el $77 \%$ de la población del estrato uno tiene acceso a éste medio. En cuanto al acceso desde teléfono móvil se trata, del 2012 al 2013 la cifra incrementó 11 puntos, pasando del $23 \%$ al 32\%, algo que evidentemente nos muestra que ha continuado creciendo, y con más fuerza, dicha penetración digital móvil en los últimos tres años. Dentro de las 10 principales actividades realizadas desde un móvil con internet en 2012, enviar y recibir correos electrónicos ocupaba el primer lugar con el $84 \%$, en segunda posición se encuentra el uso de redes sociales con un $88 \%$. El mismo estudio resalta que los jóvenes utilizan internet para estar en las redes sociales entre los 15 y 17 años, mayoritariamente.

Las cifras anteriores denotan el nivel de trascendencia que tiene llevar a cabo una campaña no convencional de concientización y promoción-prevención del consumo de tabaco. Los jóvenes, están conectados en todo momento a sus dispositivos móviles y es ahí dónde se debe intentar llamar su atención. Se nos presenta entonces un panorama móvil que muestra un nuevo paradigma, donde vivir conectado a los dispositivos como smartphones y tabletas se ha vuelto muy común "se está produciendo un fenómeno de identificación con el dispositivo pocas veces visto anteriormente en la historia de la tecnología, convirtiéndose los terminales en verdaderas extensiones de nosotros mismos". (Soro et al., 2012, p.204)

Para colonizar estos nuevos territorios, diversos desarrolladores móviles han creado una serie de aplicativos para dejar de fumar (ExFumador, Mi último Cigarrillo, QuitNow!, etc.). Estas nuevas plataformas buscan generar conciencia en los consumidores de tabaco, sin embargo, pocas veces son conscientes de los postulados necesarios para interesar a los jóvenes en las políticas de prevención y promoción. Para solucionar esta dificultad, la (Global Tobacco Free Kids, 2007) enlista una serie de postulados necesarios que deben incluir dichas campañas de comunicación:

- Usar una combinación de mensajes sobre "por qué dejar de fumar" y "cómo dejar de fumar" para motivar a los fumadores a intentar dejar de fumar. En la primera premisa los mensajes deben ser impactantes sobre las consecuencias del consumo de tabaco y mostrar emociones negativas 
(enojo, pérdida, tristeza, culpa, temor) que insten a los fumadores a realizar un intento para dejar de fumar ahora.

- En la segunda, los mensajes deben transmitir apoyo y ser positivos, enfatizar la disponibilidad de recursos para dejar de fumar y darles a los fumadores la esperanza de que pueden lograrlo.

- Usar testimonios. El sentimiento de identificación y empatía con otros casos de tabaquismo puede resultar muy efectivo, ya que las personas pueden caer en cuenta de la gravedad de su problema viéndolo frente a ellos y lograr la persuasión.

- Crear conciencia de que alguien puede ayudarlos a salir de su situación. En Estados Unidos, ClearWay MinnesotaSM, creó una línea telefónica de apoyo para los fumadores interesados en dejar de fumar.

- Resaltar que dejar de fumar es un proceso que puede incluir varios intentos.

\section{Metodología de la investigación}

El trabajo de investigación que abarcó el ejercicio de este trabajo desarrolló un componente contextual, un componente tecnológico, otro comunicativo, otro de salud y otro psicológico. Por consiguiente, el trabajo de revisión teórica y el diseño metodológico se fundamentó en la comprensión comunicativa en salud, puesto que es importante comprender la manera en la que se deben trabajar las campañas de promoción y prevención en salud en los espacios públicos digitales escolares, ya que se avizora una utilidad de este lugar de transmisión, difusión e intercambio de sujetos temáticos de conversación.

Se eligió un corpus compuesto por estudiantes de los últimos dos grados de la educación secundaria en Colombia, es decir, estudiantes de grado 10 y 11 , de 3 instituciones educativas de la ciudad de Pereira Colombia, contando con una muestra de 232 estudiantes de las instituciones educativas:

Tabla 1: corpus test cercanía al tabaco.

\begin{tabular}{|l|l|l|l|l|l|}
\hline \multicolumn{2}{|c|}{} & Frecuencia & Porcentaje & $\begin{array}{l}\text { Porcentaje } \\
\text { válido }\end{array}$ & $\begin{array}{l}\text { Porcentaje } \\
\text { acumulado }\end{array}$ \\
\hline Válido & Leningrado & 27 & 11.6 & 11.6 & 11.6 \\
\cline { 2 - 6 } & Liceo Taller San Miguel & 58 & 25.0 & 25.0 & 36.6 \\
\cline { 2 - 6 } & Luis Carlos González & 147 & 63.4 & 63.4 & 100.0 \\
\cline { 2 - 6 } & Total & 232 & 100.0 & 100.0 & \\
\hline
\end{tabular}

Fuente: Elaboración propia 
Páez V, J.E. (2019). Prevenir el consumo de tabaco en el ciberespacio escolar de la ciudad de Pereira-Colombia. Plumilla Educativa, 24(2), 85-107. DOI

Estos tres colegios se seleccionaron de manera intencional, después de contar con el acompañamiento de la Secretaría de Salud de Risaralda, pues se detectó que tenían características distintivas entre ellos que permitirían un mejor ejercicio de comparación. Se eligió un colegio de carácter privado y de estudiantes en su mayoría de clase alta, un colegio de carácter público y de estudiantes de clase media, así como un colegio de carácter público y de estudiantes de clase baja, con el fin de hacer un ejercicio de acompañamiento, capacitación y aplicación de instrumentos investigativos.

Después de seleccionada la muestra se procedió inicialmente a la aplicación de 232 encuestas (tituladas pre-test) para conocer la actitud de los jóvenes con respecto al tabaco y los estados de contemplación que poseen los que ya tienen alguno tipo de consumo.

Seguido a esta revisión previa de los estudiantes, se procedió a la aplicación de 3 grupos focales, a los cuales se les aplicaron instrumentos distintos para poder evaluar el resultado final de las intervenciones, mediante grupos control y experimento en los colegios, que finalmente pudieran entregar los resultados necesarios.

Los grupos focales se hicieron con muestras representativas de cada colegio, elegidas después de un proceso aleatorio simple, que permitió la aplicación de los siguientes instrumentos:

- Grupo focal 1 (control): En el colegio 1 se aplicó una serie de conferencias sobre el consumo de tabaco y se entregó material publicitario tradicional, con el fin de poder evaluar la efectividad de las campañas tradicionales

- Grupo focal 2 (control): En el colegio 2 se aplicó un taller de fotografía participativa titulado Photoboy, que nos permitió utilizar los medios sociales para la difusión del material entregado: especialmente Facebook.

- Grupo focal 3 (experimento): En el colegio 3 se aplicaron los dos métodos descritos anteriormente con el fin de evaluar su efectividad y revisar la pertinencia de las dos propuestas elaboradas.

Cabe aclarar que para los grupos focales 2 y 3 se eligió la fotografía participativa como estrategia de construcción propia del mensaje y como instrumento gráfico que a través de la imagen puede poner a circular contenidos mediáticos en ciberespacios escolares (Rabadán y Contreras, 2014)

Después de la aplicación de estos instrumentos se realizó la aplicación de una encuesta final titulada (post-test), la cual permitió corroborar el cambio de actitudes y estado de contemplación de los jóvenes después de participar en una campaña tradicional, en una alternativa o en las dos al mismo tiempo. 


\section{Resultados de la investigación}

Después de aplicar los pretest a los estudiantes de los 3 colegios elegidos se encontró que el $45,4 \%$ de los estudiantes tienen miembros de su núcleo familiar que fuman, un resultado muy alto, sin embargo, fue más preocupante el alto número de amigos cercanos que tienen ese hábito, cerca del $40.2 \%$. Podemos afirmar entonces que los jóvenes si tienen un contacto cercano con la problemática, a pesar de no ser fumadores, ya que el $84 \%$ de los estudiantes manifestó que no fuma ni nunca ha fumado, sin embargo, el 16\% restante que fuma, afirma que lo hace en su gran mayoría por experimentar nuevas sensaciones, tal cual se ve en la siguiente tabla:

Tabla 2: resultados pre-test- cercanía al tabaco.

\begin{tabular}{|c|c|c|c|c|c|}
\hline \multicolumn{6}{|c|}{$\begin{array}{l}\text { 1. Elija entre las siguientes opciones las que mejor describan su situación con respecto a la } \\
\text { cercanía al tabaco (pueden marcar varias opciones) }\end{array}$} \\
\hline & & Frecuencia & Porcentaje & $\begin{array}{l}\text { Porcentaje } \\
\text { válido }\end{array}$ & $\begin{array}{l}\text { Porcentaje } \\
\text { acumulado }\end{array}$ \\
\hline \multirow[t]{6}{*}{ Válido } & & 58 & 25.0 & 25.0 & 25.0 \\
\hline & $\begin{array}{l}\text { Alguno o algunos de los } \\
\text { miembros de mi núcleo } \\
\text { familiar fuman }\end{array}$ & 79 & 34.1 & 34.1 & 59.1 \\
\hline & $\begin{array}{l}\text { En mi casa hay personas } \\
\text { que fuman }\end{array}$ & 24 & 10.3 & 10.3 & 69.4 \\
\hline & Mi pareja fuma & 1 & 0.4 & 0.4 & 69.8 \\
\hline & $\begin{array}{l}\text { Uno o varios de mis } \\
\text { amigos cercanos fuman }\end{array}$ & 70 & 30.2 & 30.2 & 100.0 \\
\hline & \begin{tabular}{|l|} 
Total \\
\end{tabular} & 232 & 100.0 & 100.0 & \\
\hline
\end{tabular}

Fuente: Elaboración propia

Estos resultados permitieron evidenciar que el público objetivo no es un público fumador, sin embargo, tiene muchas probabilidades de convertirse en fumador debido al consumo recurrente en su círculo social cercano. Según autores como Flórez (1996), Dallery et al. (2015) y Torres (1998), el consumo de tabaco se da por círculos cercanos enfocados en la "moda" que genera la experimentación juvenil que se ve mediante la repetición de pares, en este caso amigos. Los resultados para este caso, evidencian que un $45,5 \%$ de los estudiantes afirmaron que definitivamente no aceptarían un cigarrillo por parte de sus compañeros, pero dejaron abierta la posi- 
bilidad de consumo en un $55 \%$ como se puede evidenciar en la siguiente gráfica comparativa.

\section{Figura 1. Probabilidad de consumo general previo a la estrategia de internvención}

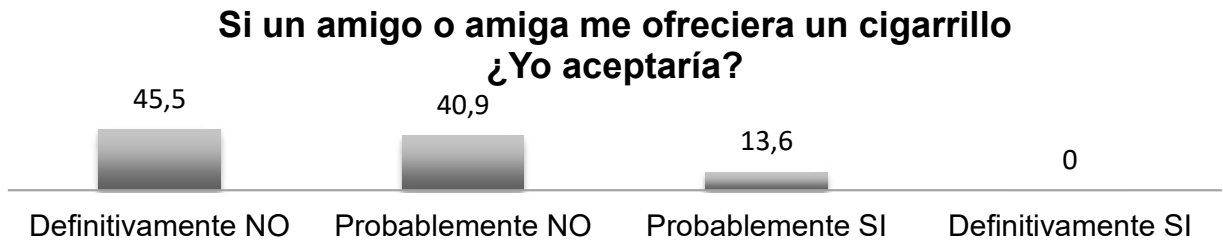

Fuente: propio

Después de aplicados estos pretest, el proyecto de investigación realizó una serie de grupos focales con los estudiantes para evidenciar cómo las estrategias tradicionales y alternativas podrían cambiar radicalmente esta percepción sobre el tabaco y su consumo. Es por esta razón que se diseñó la aplicación de estrategias tradicionales para un grupo (Colegio Leningrado), una estrategia alternativa en el ciberespacio (Colegio Luis Carlos González) y una estrategia mixta (Colegio Liceo Taller San Miguel)

En el colegio Leningrado la estrategia tradicional consistió en llevar el conversatorio de cáncer infantil, liderado por un profesional de Oncólogos de Occidente, donde se les habló de los riesgos de consumir tabaco, así como la estrategia: La Decisión está en tus manos, que tiene al doctor feliz como su principal elemento de prevención, al igual que la entrega de folletos y material publicitario tradicional, como se puede ver en la siguiente imagen.

\section{Figura 2. Grupo focal tradicional (Leningrado)}
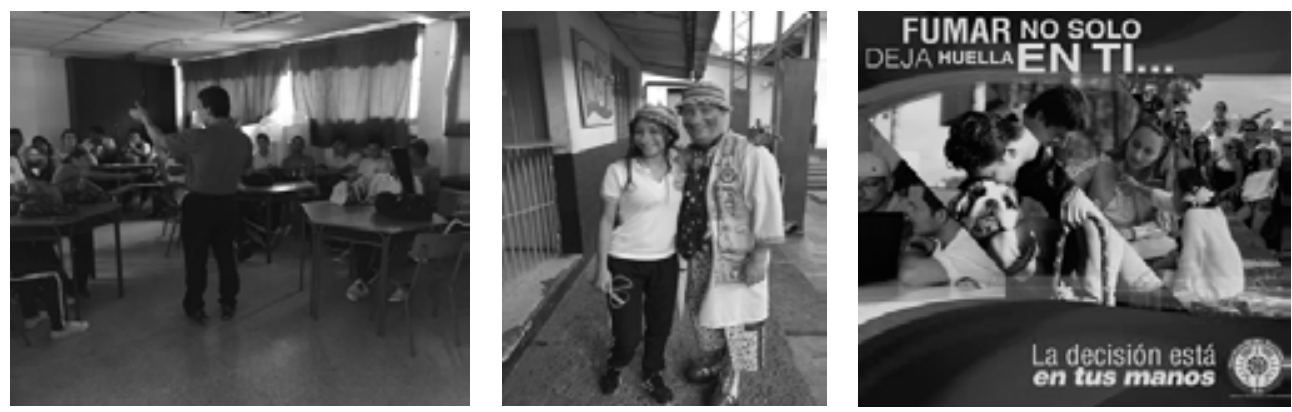

Fuente: propio 
En el colegio Luis Carlos González, la estrategia fue alternativa y utilizó la fotografía participativa como elemento capaz de transmitir mensajes de prevención del consumo de tabaco. Según Rabadán y Contreras (2014), el uso de la fotografía participativa es importante porque permite que los investigados evidencien su contexto, su sitio en el mundo y su entorno, a través de la toma de fotografías que evidencian una serie de características sociales y de múltiples desafíos. Teniendo en cuenta lo anterior, se llevó a que los estudiantes participaran en un taller de fotografía participativa titulado Photoboy, en donde les entregaron los conocimientos necesarios para realizar fotografías profesionales correctas y en donde finalmente se les pidió que realizaran una fotografía que evidenciara la cercanía que tenían con el tabaco y la subieran a redes sociales para ver la reacción de sus compañeros en dicho ciberespacio.

Esta estrategia alternativa buscó reconocer la importancia de los estudiantes como miembros del ciberespacio y como ciberciudadanos capaces de consumir, pero al mismo tiempo construir mensajes de promoción y prevención, como lo podemos ver en las siguientes imágenes.

\section{Figura 3. Grupo focal alternativo (Luis Carlos González)}
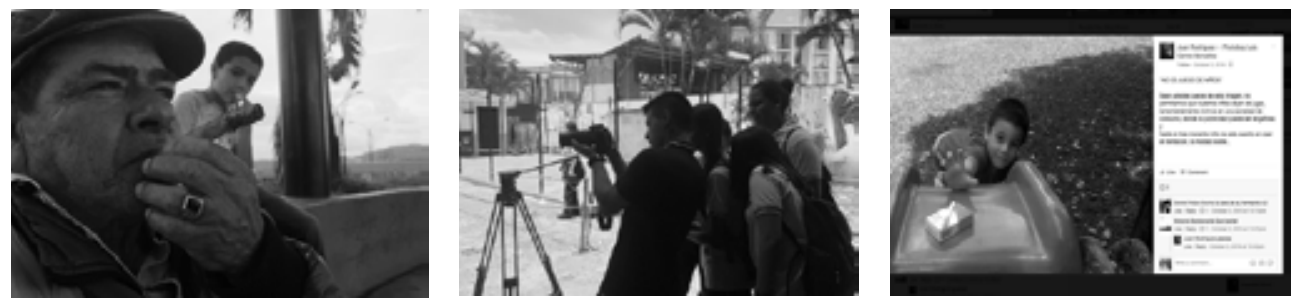

Fuente: propio

La presente estrategia generó un impacto en los usuarios de las redes sociales, pues evidenció una cercanía de la temática con los estudiantes en su ciberespacio, y es aquí donde se hace relevante utilizar los elementos participativos a la hora de construir el mensaje comunicativo de las campañas, pero, sobre todo, aumentar los comentarios positivos de los mismos utilizando herramientas de uso constante como lo pueden ser Facebook u otras redes sociales digitales. Estos resultados evidencian además la importancia de reflexionar sobre los usos sociales de los medios de comunicación, tal cual se presentó anteriormente (Lull, 1980). Esta estrategia evidencia la necesidad de generar usos relacionales y no solamente estructurales, los cuales permi- 
tan dar la voz a los estudiantes, con el fin de convertirlos en productores de la información, y permitirles que encuentren sentidos más amplios a este uso, por ejemplo: el de aumentar sus relaciones personales, evidentes en los grupos de Facebook donde se gestionó el concurso, o simplemente generando mejoras en su identidad personal, como se evidenció en las fotografías tomadas y la justificación entregada por los estudiantes. Un enfoque más cercano a la teoría de las gratificaciones de Mcquail (1972).

Sin embargo, el proyecto de investigación quería ver además lo que pasaba cuando se alternaban estas dos estrategias y fue ahí donde se utilizó el colegio Liceo Taller San Miguel, a quienes se les aplicaron las dos estrategias descritas anteriormente, charla de prevención, material publicitario de la campaña: la decisión está en tus manos y cursos de fotografía participativa, como se puede ver en las siguientes imágenes.

\section{Figura 4. Grupo focal mixto (Liceo Taller San Miguel)}
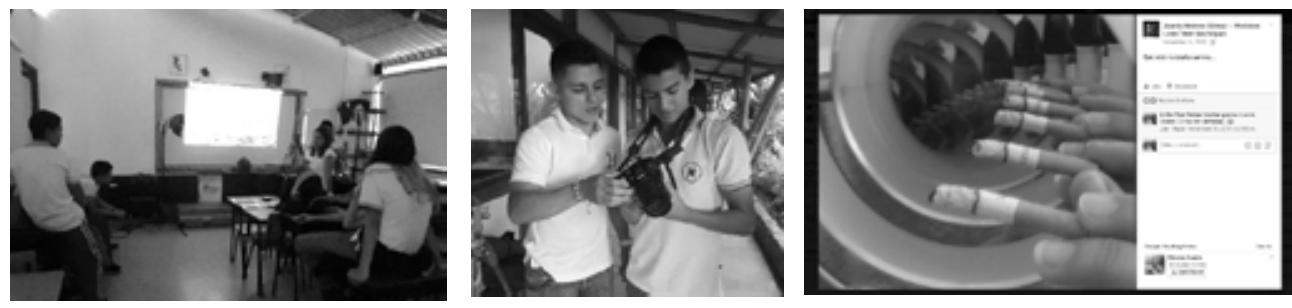

Fuente: propio

\section{Discusión}

Después de la aplicación de los 3 grupos focales se procedió a aplicar un nuevo test, en esta ocasión denominado (post-test) para evaluar la efectividad de cada una de estas estrategias en la disminución de las actitudes positivas hacia el tabaco, con el fin de poder determinar la relevancia que tienen los nuevos medios, pero sobre todo, los intercambios en el ciberespacio para los jóvenes de la ciudad de Pereira. Con estos post-test se logró determinar que la utilización de la fotografía participativa como elemento de prevención es muy funcional (Rabadán y Contreras, 2014), ya que los jóvenes consideran que hacen parte de la construcción del mensaje, pero sobre todo porque el lugar de distribución de este material es netamente comunicativo. Los estudiantes tienen la posibilidad de interactuar con la campaña que ellos mismos crean y eso disminuye sustancialmente la percepción que tienen sobre el consumo de esta sustancia, como podemos evidenciar en la siguiente gráfica. 
Figura 5. Probabilidad de consumo general posterior a la estrategia de intervención

\section{Después del grupo focal, si un amigo o amiga me ofreciera un cigarrillo ¿Yo aceptaría?}

\begin{tabular}{cccc}
73,2 & 20,1 & 6,2 & 0,5 \\
\hline Definitivamente NO & Probablemente NO & Probablemente SI & Definitivamente SI
\end{tabular}

Fuente: propio

Según los resultados, cuando los estudiantes responden de nuevo a esta pregunta, podemos ver que un $73 \%$ de ellos afirman que definitivamente NO probarían el cigarrillo si un amigo se los ofreciera, lo que evidencia la efectividad de las estrategias implementadas, pero sobre todo la disminución de dicha presión social que generan los pares, en este caso sus propios compañeros.

Sin embargo, el resultado más relevante es ver como el $80 \%$ de los estudiantes que participaron de la estrategia mixta de prevención afirman que definitivamente no probarían el cigarrillo si un amigo se los ofreciera (colegio Liceo Taller San Miguel). Lo que nos muestra que una mezcla entre estrategias tradicionales (conversatorios + material publicitario) y una estrategia alternativa (fotografía participativa en Facebook) puede ser más efectiva para prevenir el consumo. Mientras que en los colegios que recibieron solamente una de las campañas diseñadas (tradicionales y alternativas), la cifra de estudiantes que no probarían el cigarrillo ofrecido por sus compañeros sube solamente al $70 \%$, como podemos ver en la siguiente gráfica.

Figura 6. Probabilidad de consumo comparativo por colegio posterior a la estrategia de intervención

\section{Probabilidad de consumo comparativo por colegios}

- Luis Carlos González Liceo Taller San Miguel Leningrado $70,6 \quad 80,8 \quad 71,4$

\begin{tabular}{ccccccccccc} 
& 24,5 & 9,6 & 14,3 & & 4,9 & 7,7 & 14,3 & 0 & 1,9 & 0 \\
\hline Definitivamente NO & \multicolumn{2}{c}{ Probablemente NO } & \multicolumn{2}{c}{ Probablemente SI } & \multicolumn{2}{c}{ Definitivamente SI }
\end{tabular}


Páez V, J.E. (2019). Prevenir el consumo de tabaco en el ciberespacio escolar de la ciudad de Pereira-Colombia. Plumilla Educativa, 24(2), 85-107. DOI

Con estos resultados podemos afirmar entonces que las campañas tanto tradicionales como alternativas son útiles, pero la mezcla de estas dos tiene resultados más positivos, ya que la cifra de percepción negativa al consumo del tabaco sube en casi $10 \%$, logrando un $80 \%$ de disminución del consumo ofrecido por pares.

Además cabe aclarar que los estudiantes que participaron en la estrategia mixta de prevención lograron fotografías mucho más elaboradas y que representan mayor análisis de la problemática, ya que tienen un simbolismo mayor a las entregadas por el colegio Luis Carlos González, así como una técnica más elaborada, juego de luces y sombras, mejores encuadres, planimetrías y que podría circular con facilidad en cualquier campaña de promoción y prevención, además porque su difusión en ciberespacios escolares produjo mayor número de reacciones.

\section{Conclusiones}

Podríamos concluir entonces diciendo que los estudiantes disminuyen sus actitudes positivas hacia el tabaco cuando se les permite ser los creadores del mensaje. En la ciudad de Pereira Colombia los estudiantes no tienen elevadas cifras de consumo de tabaco, sin embargo, están rodeados de ambientes y personas que podrían propiciar con facilidad dicho consumo. La posibilidad de consumo es alta si se ve la influencia de los amigos en la idea de experimentación que produce el consumo de tabaco, es por esta razón que las campañas de comunicación deben atacar esa sensación de repetición de consumo entre amigos en sus mensajes

Las campañas tradicionales de prevención (conversatorios y diseño de piezas publicitarias masivas) pueden ser efectivas disminuyendo la actitud positiva hacia el consumo de tabaco, sin embargo, no utilizan la interactividad que se tiene hoy en día en los ciberespacios escolares (Benkler, 2006). Los estudiantes reaccionan positivamente a las iniciativas que involucran su mirada personal, es por esta razón que todas las campañas de prevención deberían diseñarse no por publicistas o especialistas en la comunicación, sino por los mismos actores del acto comunicativo, con el fin de generar interactividad desde el inicio de la campaña de promoción o prevención.

Los medios digitales, pero especialmente las redes sociales digitales como Facebook, son un nuevo ágora de intercambio juvenil, en donde incluir este tipo de mensajes puede ser mucho más efectivo, ya que propicia un mayor número de retroalimentaciones que los medios tradicionales (Valdez, 2016). Las estrategias de prevención del consumo de tabaco de- 
berían entonces alternar sus estrategias en diferentes canales de difusión, pero, sobre todo, con diferentes puntos de vista a la hora de construir sus contenidos (Scolari, 2013).

Finalmente, el consumo del tabaco podría verse disminuido y la posible actitud de consumo, si se le enseña al estudiante un universo diferente que pueda ser más atractivo que el tabaco, en el caso de esta investigación la fotografía, como elemento capaz de pintar la realidad de cada estudiante mediante la luz, pero, sobre todo, proyectar un futuro alejado de este tipo de prácticas nocivas para la salud. 
Páez V, J.E. (2019). Prevenir el consumo de tabaco en el ciberespacio escolar de la ciudad de Pereira-Colombia. Plumilla Educativa, 24(2), 85-107. DOI

\section{Referencias}

Anzieu, D., Martin, J. Y., \& Zimmermann, S. V. (1997). La dinámica de los grupos pequeños. Madrid: Biblioteca Nueva.

Beck, U. (1998). La sociedad del riesgo: hacia una nueva modernidad. Madrid: Paidós ibérica

Benkler, Y. (2006). The wealth of networks: How social production transforms markets and freedom. New Haven: Yale University Press.

Caylà, Joan A et al. (2016). La infección por virus Zika: una nueva emergencia de salud pública con gran impacto mediático. Madrid, España. Gaceta Sanitaria [online]. 2016, v. 30, n. 6 Accedido 12 Setiembre 2019, pp. 468471. Disponible en: https://doi.org/10.1016/j.gaceta.2016.05.015.

CCLAT Convención general ley antitabaco Colombia (2009) Ley 1335 de 2009. (Consultado en línea el 15 de marzo de 2017) en la dirección: http:// www.alcaldiabogota.gov.co/sisjur/normas/Norma1.jsp?i=36878

Champagne, P. (1995). Opinion publique" et débat public. L'espace public et l'emprise de la communication. Grenoble: Ed. Ellug

Comisión Nacional de Televisión. (2010). Resolución 2010-380-001136-4, Por medio de la cual se reglamenta la emisión de mensajes institucionales de prevención contra el consumo de tabaco y sus derivados en el servicio público de televisión, y se modifican algunas disposiciones contenidas en la Resolución 290 de 2002, en cumplimiento de lo dispuesto en la Ley 1335 de 2009. Bogotá, Colombia. Comisión Nacional de Televisión.

Comité National Contre le Tabagisme. (2016). Les campagnes de communication qui font peur sont-elles efficaces ?. (Consultado en línea el 18 de marzo de 2016) en la dirección: http://www.cnct.fr/informer-etalerter-13/2-focus-sur-les-campagnes-anti-tabac-diffusees-dans-lesmedias-2-126.html

Congreso de la República de Colombia. (2009). Ley 1335 de 2009. Julio 21. Disposiciones por medio de las cuales se previenen daños a la salud de los menores de edad, la población no fumadora y se estipulan políticas públicas para la prevención del consumo del tabaco y el abandono de la dependencia del tabaco del fumador y sus derivados en la población colombiana. Bogotá: Congreso de la República de Colombia.

Dallery, Jesse; Jarvis, Brantley; Marsch Lisa, Xie, (2015) Haiyi. Mechanisms of change associated with technology-based interventions for substance use. Drug and alcohol dependence, vol. 150, 14-23 
DANE (2013). Total suscriptores internet en Pereira. (Consultado en línea el día 14 de marzo de 2017), en la dirección: http://estrategiaticolombia. co/estadisticas/stats. . php? \&pres=content $\&$ jer $=3 \&$ cod=66001\&id=34\#TTC

Entman, R. M. (1993). Framing: Toward clarification of a fractured paradigm. Journal of communication, 43(4), 51-58.

Flórez R. (1996). Dinámica del desarrollo cognitivo individual. En: Hacia una pedagogía del conocimiento. Bogotá: Mc. Graw Hill. 62-74

Fraser, N. (1990). Rethinking the public sphere: A contribution to the critique of actually existing democracy. Social text, (25/26), 56-80.

Global Tobacco Free Kids, (2007). Paquete de herramientas para el desarrollo de campañas. (Consultado en línea el día 14 de marzo de 2017) en la dirección: http://global.tobaccofreekids.org/es/resources/

Habermas, J., i Ramió, J. R., Domènech, A., \& Grasa, R. (1981). Historia y crítica de la opinión pública: la transformación estructural de la vida pública (pp. 1-171). Barcelona: Editorial Gustavo Gili.

León, L. M. T., \& Bonilla, N. J. V. (2011). Conocimientos, actitudes y prácticas sobre ambientes libres de humo, actividad física y dieta saludable en tres colegios de Pereira. Pereira, Colombia. Revista Médica de Risaralda, 17(1).

Lull, J. (1980). The social uses of television. Human communication research, 6(3), 197-209.

Maletzke, G. (1976). Psicología de la comunicación social. CIESPAL. Centro Internacional de Estudios Superiores de Periodismo para América. Quito: Editorial Qipos.

McQuail, D., Blumler, J. G., \& Brown, J. R. (1972). The television audience: A revised perspective. Media studies: A reader, 271, 284.

Mosquera, M. (2003). Comunicación en salud: conceptos, teorías y experiencias. La iniciativa de la comunicación. Comunicación y medios para el desarrollo de América Latina y el Caribe (52). Recuperado de: http:// www.comminit.com/la/drum_beat_52.html

Mosquera, J., \& Mateus, J. C. (2003). Conocimientos, actitudes y prácticas sobre métodos de planificación familiar, VIH-SIDA y el uso de los medios de comunicación en jóvenes. Colombia Médica, 34(4), 206-212.

$\mathrm{NCl}$, National Cancer Institute. (1991). Cancer statistics review, 1973-1989. Estados Unidos. B. Miller (Ed.) National Cancer Institute.

Pardo, C. y Piñeros, M. (2010). Consumo de tabaco en cinco ciudades de Colombia, Encuesta Mundial de Tabaquismo en Jóvenes, 2007. Biomédica, 30(4). 
Pérez, M. Á. y Pinzón-Pérez, H. (2005). Uso del tabaco entre los jóvenes colombianos. Retos para los profesionales en salud pública. Revista Científica Salud Uninorte, vol. 21. Recuperado de: http://rcientificas.uninorte.edu.co/index.php/salud/article/ viewArticle/4112/5675

Rabadán Crespo, Á. V., \& Contreras Pulido, P. (2014). La Fotografía Participativa en el contexto socio-educativo con adolescentes. comunicación y Hombre, (10).

Rieder, B. (2012). The refraction chamber: Twitter as sphere and network. First Monday, 17(11).

Salván, E. J., \& Izquierdo, M. J. (2014). Los errores de comunicación en la crisis del ébola. Madrid, España. Cuadernos de periodistas: revista de la Asociación de la Prensa de Madrid, (29), 90-99

Scolari, C. A. (2013). Narrativas transmedia: cuando todos los medios cuentan. Barcelona: Editorial Deusto.

Scheufele, D. A., \& Tewksbury, D. (2006). Framing, agenda setting, and priming: The evolution of three media effects models. Journal of communication, 57(1), 9-20.

Soro Jordán, D., Estrade Nieto, J. M., \& Hernández Dauder, M. A. (2012). Marketing digital: marketing móvil, SEO y analítica web. Madrid, España. Anaya Multimedia-Anaya Interactiva.

Suau-Gomila, G., Percastre-Mendizabal, S., Palà, G., y Pont, C. (2017) Análisis de la comunicación de emergencias en Twitter. El caso del Ébola en España. En: Sierra, J. \& Liberal, S. Uso y Aplicación de las Redes Sociales en el Mundo Audiovisual y Publicitario. (119-130). Madrid: McGrawhill Education.

Torres, J. (1998) Globalización e interdisciplinariedad: el currículo integrado. Madrid: Ediciones Morata.

Valdez, J. P. (2016). Estructura y contenido de la twitteresfera política colombiana durante las elecciones legislativas de 20141. Pereira, Colombia. Revista Textos y Sentidos, (13), 27-47.

WHO, World Health Organization. (2007). International Classification of Functioning, Disability, and Health: Children \& Youth Version: ICF-CY. World Health Organization.

WHO, World Health Organization. (1986). Ottawa charter. Geneva: World Health Organization.

Recibido: 29 de julio de 2019.

Aceptado: 30 de septiembre de 2019. 